\title{
Emodin Promotes Autophagy and Prevents Apoptosis in Sepsis-Associated Encephalopathy through Activating BDNF/TrkB Signaling
}

\author{
Li-Li Gao $^{a} \quad$ Zhi-Hao Wang ${ }^{b} \quad$ Yu-Hang Mu Zuo-Long Liu $^{a} \quad$ Li Pang $^{\text {a }}$ \\ aDepartment of Emergency ICU, The First Hospital of Jilin University, Changchun, China; ${ }^{b}$ Department of \\ Geriatrics, The First Hospital of Jilin University, Changchun, China
}

\section{Keywords}

Emodin · Sepsis-associated encephalopathy · BDNF/TrkB signaling · Apoptosis · Autophagy

\begin{abstract}
Objective: Sepsis-associated encephalopathy (SAE) is a severe and common complication of sepsis and can induce cognitive dysfunction and apoptosis of neurons and neuroinflammation. Emodin has been confirmed to have anti-inflammatory effects. Thus, we sought to investigate the role of Emodin in SAE. Methods: The cecal ligation and puncture (CLP) method was used for the establishment of SAE in mice model. For treatment of Emodin, intraperitoneal injection of $20 \mathrm{mg} / \mathrm{kg}$ Emodin was performed before the surgery. The Morris water maze and open field tests were carried for measurement of cognitive dysfunction. Hematoxylin and eosin staining was for histological analysis of hippocampus. Cell apoptosis of hippocampus neurons was measured by TUNEL staining. Pro-inflammatory and anti-inflammatory cytokines in hippocampus tissue homogenate were evaluated by ELISA. BDNF/TrkB signaling-related proteins (TrkB, p-TrkB, and BDNF), autophagy-related proteins (LC3 II/I and Beclin-1), and apoptosis-related proteins (Bax, $\mathrm{Bcl}-2$, and cleaved caspase-3) were detected by Western blotting. Results: Emodin
\end{abstract}

\begin{tabular}{ll}
\hline karger@karger.com & (c) 2021 The Author(s). \\
www.karger.com/pat & Published by S. Karger AG, Basel \\
& $\begin{array}{l}\text { This is an Open Access article licensed under the Creative Commons } \\
\text { Attribution-NonCommercial-4.0 International License (CC BY-NC) } \\
\text { (http://www.karger.com/Services/OpenAccessLicense), applicable to } \\
\text { the online version of the article only. Usage and distribution for com- } \\
\text { mercial purposes requires written permission. }\end{array}$
\end{tabular}

significantly inhibited apoptosis and induced autophagy in hippocampal neurons of CLP-treated mice. In addition, Emodin significantly ameliorated CLP-induced cognitive dysfunction and pathological injury in mice. Meanwhile, Emodin notably inhibited CLP-induced inflammatory responses in mice via upregulation of BDNF/TrkB signaling, while the effect of Emodin was partially reversed in the presence of K252a (BDNF/TrkB signaling inhibitor). Conclusion: Emodin significantly inhibited the progression of SAE via mediation of BDNF/TrkB signaling. Thus, Emodin might serve as a new agent for SAE treatment.

(c) 2021 The Author(s).

Published by S. Karger AG, Basel

\section{Introduction}

Sepsis-associated encephalopathy (SAE) is defined as diffuse brain dysfunction without direct central nervous system infection in patients with sepsis, and SAE is one of the most common complications of sepsis [1,2]. SAE may induce severe cognitive dysfunction and is a risk factor for patients' death $[3,4]$. The major risk factors for SAE include surgical and bacterial infection [5]. In addition, the progression of SAE usually leads to the increase of apoptosis and inhibition of autophagy in neurons [6]. Now- 
adays, the main treatment of SAE is drug therapy [7], while the effect remains very limited. Thus, it is urgent to find new treatment methods for SAE.

Emodin (1, 3, 8-trihydroxy-6-methylanthraquinone) is an extract from Rhubarb, which has been found to show anti-inflammatory effect $[8,9]$. It was reported that Emodin showed anti-inflammatory effects in LPS-induced RAW264.7 cells [10]. Recent studies also found Emodin inhibited inflammatory response in severe acute pancreatitis and might attenuate NLRP3 inflammasome activation $[11,12]$. Besides, Emodin also inhibited cell apoptosis to alleviate the inflammation [13]. On the other hand, Emodin is involved in autophagy and cognitive dysfunction during the neurons' injury. For example, Emodin could inhibit aggregation of amyloid $\beta$ peptide 1-42 and improve cognitive deficits in Alzheimer's disease [14]. Moreover, Emodin could alleviate sepsis-induced injury via inducing autophagy $[15,16]$. Meanwhile, SAE progression could cause the inhibition of autophagy [17]. Based on the background, it can be speculated that Emodin can regulate the progression of SAE.

It has been reported that inhibition of BDNF/TrkB signaling may lead to the inflammation [18]. In addition, Ma et al. [19] found that $\alpha$-synuclein is involved in manganese-induced spatial memory and synaptic plasticity impairments via regulation of BDNF/TrkB signaling. This result suggested that downregulation of BDNF/TrkB signaling may contribute to the neuronal injury. Moreover, inactivation of $\mathrm{BDNF} / \mathrm{TrkB}$ signaling could result in promotion of cell apoptosis, inhibition of cell autophagy, and upregulation of inflammatory responses $[9,14,17]$. Based on these data, it can be supposed that BDNF/TrkB signaling might act as a key mediator in progression of SAE. Nevertheless, the correlation between Emodin and $\mathrm{BDNF} / \mathrm{TrkB}$ signaling is not clear.

In the present study, we aimed to investigate effects of Emodin on SAE mice and the underlying molecular mechanisms. We demonstrated for the first time that Emodin improved cognitive dysfunction, suppressed hippocampal neurons' injury, inhibited inflammatory response and cell apoptosis, and promoted cell autophagy through activation of BDNF/TrkB signaling in SAE mice. This study might provide experimental basis for Emodin in treatment of SAE.

\section{Methods and Materials}

\section{Animals and Treatment}

A total of 72 male BALB/C mice (6-8 weeks, 18-22 g) were purchased from SJA Laboratory Animal Co. (Hunan, China). All animals were housed in micro-isolator cages with free access to food and water according to the Guide for the Care and Use of Laboratory Animals. In particular, any effort was put to avoid unnecessary pain of the animals. The whole study was approved by the Experimental Animal Ethics Committee at the First Hospital of Jilin University.

All animals were divided into the following groups: control group, sham group, cecal ligation and puncture (CLP) group, CLP + Emodin group, sham + Emodin group, CLP + Emodin + K252a group, CLP + Emodin + DMSO group, and CLP + Emodin + 3-MA group. For all animal experiments, each group included 9 mice.

For establishment of SAE in vivo model, mice were treated with CLP method as reported previously [20]. Briefly, mice were anaesthetized with pentobarbital sodium $(50 \mathrm{mg} / \mathrm{kg})$ [21] by intraperitoneal injection and the cecum was ligated for 2 days post. In addition, mice received the same surgical treatment without cecal ligation sham group. No mouse was dead during in vivo model establishment.

For treatment of Emodin, mice received intraperitoneal injection of $20 \mathrm{mg} / \mathrm{kg}$ Emodin (Sigma-Aldrich, St. Louis, MO, USA) at 30 min before the surgery. For inhibition of BDNF/TrkB signaling, mice received intraperitoneal injection of $25 \mathrm{mg} / \mathrm{kg} \mathrm{BDNF} / \mathrm{TrkB}$ signaling inhibitor K252a (Sigma-Aldrich) at $30 \mathrm{~min}$ before the surgery. Meanwhile, mice in CLP + Emodin + 3-MA group were intraperitoneally injected with 3-MA (MedChemExpress; $30 \mathrm{mg}$ / $\mathrm{kg}$ ). The control groups received the same volume of DMSO.

\section{Behavior Experiments}

For measurement of cognitive dysfunction, the Morris water maze (MWM) and open field tests were carried [22]. Briefly, for MWM test, mice were placed in a circular pool with water $(24 \pm$ $1^{\circ} \mathrm{C}$ ). A transparent platform was placed with surface $2 \mathrm{~cm}$ below the water. The mice were placed facing the wall to locate the submerged platform in $60 \mathrm{~s}$. All mice activity was tracked and could be displayed by the computer software (MWM; Yihong Technology Co., Ltd, Wuhan, China). The escape latency to find the platform and the frequency of target platform crossings were measured in different mice groups. For the open field test, mice were placed in an open field reactor with black inwall, which was under monitoring by a camera. The mice movement distance, track, and moving frequency were all recorded.

\section{Tissue Collection}

Animals were sacrificed with anesthesia by pentobarbital (150 $\mathrm{mg} / \mathrm{kg}$ ) [23] after the MWM and open field tests. The hippocampuses of mice were collected. There are 6 mice of each group made for paraffin section, fixed with 4\% paraformaldehyde, immersed in $3 \% \mathrm{H}_{2} \mathrm{O}_{2}$, and cut into 4 - $\mu \mathrm{m}$-thick slices. Moreover, there are 3 mice of each group homogenated, which were prepared in ice-cold phosphate-buffered saline ( $\mathrm{pH}$ 7.4). The homogenates were centrifuged ( $200 \mathrm{~g}, 10 \mathrm{~min})$, and the supernatant was collected separately.

\section{H\&E Staining}

Paraffin sections (after dewaxed and dehydrated) were routinely washed with distilled water, stained with Mayer hematoxylin (Beyotime, Shanghai, China) at room temperature for $5 \mathrm{~min}$, and flushed under tap water for $1 \mathrm{~min}$. Next, the sections were re-stained with $1 \%$ eosin solution for $1 \mathrm{~min}$. Histopathological changes were assessed by an optical microscope (Olympus, Tokyo, Japan). 
TUNEL Staining

TUNEL staining was used for measurement of cell apoptosis of hippocampus neurons. Briefly, after dewaxed and dehydrated, paraffin sections were stained with a TUNEL assay kit (ab206386; Abcam, Cambridge, MA, USA) according to the manufacturer's instructions. An optical microscope was used to take the photomicrographs.

Enzyme-Linked Immunosorbent Assay

Inflammatory factors of IL- $1 \beta$, IL- 6 , TNF- $\alpha$, TGF- $\beta$, IL- 4 , and IL-10 in hippocampus tissue homogenate were evaluated by using commercial enzyme-linked immunosorbent assay (ELISA) kits strictly according to manufactures' instructions (IL-1 $\beta$ ab197742 Abcam, IL-6 ab100713 Abcam, TNF-a ADI-900-047 Enzo Life Sciences, TGF- $\beta$ ab100647 Abcam, IL-4 ab215089 Abcam, and IL-10 ab185986 Abcam).

\section{Western Blotting}

Proteins were extracted from tissue homogenate using radioimmunoprecipitation assay buffer (Vazyme Biotech Co., Ltd, Nanjing, China). The total proteins were quantified by BCA kit (Beyotime). The extracted proteins were subjected to SDS-PAGE, transferred to polyvinylidene difluoride membranes, and blocked by $5 \%$ nonfat milk at room temperature for $1 \mathrm{~h}$. After incubation with primary antibodies (Abcam) anti-TrkB (ab187041, 1:5,000), anti-p-TrkB (ab229908, 1:1,000), anti-BDNF (ab108319, 1:1,000), anti-LC3B (ab192890, 1:2,000), anti-Beclin-1 (ab210498, 1:1,000), anti-Bax (ab182733, 1:2,000), anti-Bcl-2 (ab182858, 1:2,000), anti-cleaved caspase-3 (ab214430, 1:5,000), anti- $\beta$-actin (ab8226, 1:1,000), and anti-GAPDH $(\mathrm{ab} 8245,1: 1,000)$ at $4^{\circ} \mathrm{C}$ overnight, samples were incubated with a secondary antibody (IgG, ab96879, 1:2,000) at $37^{\circ} \mathrm{C}$ for $1 \mathrm{~h}$. Finally, membranes were visualized with the enhanced chemiluminescent detection system (GE Healthcare, Piscataway, NJ, USA). The densities of blots were normalized to GAPDH.

\section{Statistical Analysis}

All data were presented as the mean \pm SD, using SPSS 20.0 (IBM, Armonk, NY, USA) for data analysis. Two groups were compared using Student's $t$ test, and multiple groups ( $>2$ groups) were compared using 1-way ANOVA and Tukey's post hoc tests. $p<0.05$ was considered statistically significant. All experiments were repeated 3 times.

\section{Results}

Emodin Improved Cognitive Dysfunction and Suppressed Pathological Injury of Hippocampal Neurons in SAE Mice

Since the mechanism by which Emodin alleviates the symptom of SAE remains unclear, we sought to investigate the effect of Emodin on SAE in vivo. First, we evaluated the effects of Emodin on cognitive dysfunction of SAE mice. MWM test was conducted in order to evaluate learning, memory capacity as well as the spatial positioning ability of the rats [24]. In addition, escape latency means the time it took for the rat to find the platform hidden under the water, which represents the memory capac-

Emodin Improves Sepsis-Associated Encephalopathy ity of rats [24]. Thus, the MWM test was performed. As shown in Figure 1a, the MWM test showed that the escape latency of mice treated by CLP method significantly increased compared with the sham and control mice, as well as the sham mice treated with Emodin (all $p<0.05)$. Meanwhile, no significant difference was found among control, sham, and sham mice treated with Emodin, indicating Emodin did not influence cognitive dysfunction in normal mice. However, when treated with Emodin, the escape latency remarkably decreased in CLP mice $(p<0.05)$. Similarly, it was also observed that in MWM test, the frequency for mice crossing platform quadrant was markedly lower in CLP group compared with the sham as well as control groups ( $p<0.05$, Fig. 1b). And when treated with Emodin, the frequency significantly increased compared with CLP mice $(p<0.05)$. In open field test, it was also found that the movement distance of CLP mice was markedly decreased, while treatment of Emodin significantly rescued the effects by CLP (all $p<0.05$, Fig. 1c).

Besides, there was no obvious pathological damage in the CA1 structure of the hippocampus of control, sham, and sham + Emodin group, and the cell structure was complete. However, the hippocampal tissue had more severe pathological damage, with shrinking neuronal nuclei, incomplete morphology and structure, and disordered cell arrangement after CLP treatment (Fig. 1d). When treated with Emodin, the damaged cellular structure by CLP was partly recovered. All these results suggested that Emodin improved cognitive dysfunction and pathological injury of hippocampal neurons in SAE mice.

\section{Emodin Inhibited Levels of Inflammatory Factors and} Activated BDNF/TrkB Signaling in SAE Mice

Emodin has been confirmed to have anti-inflammatory effect [25]. Thus, in order to confirm the anti-inflammatory effect of Emodin on progression of SAE, inflammatory factors were detected in SAE mice. When treated by CLP method, the levels of pro-inflammatory factors (IL-1 $\beta$, IL-6, and TNF- $\alpha$ ) were all significantly upregulated, while the levels of anti-inflammatory factors (IL-10, IL-4, and TGF- $\beta$ ) were all downregulated, compared with the control or sham groups, as well as sham group treated by Emodin (all $p<0.05$, Fig. 2a). However, Emodin significantly decreased the levels of pro-inflammatory factors and increased the levels of anti-inflammatory factors in CLP mice (all $p<0.05$, Fig. 2a). In contrast, both protein levels of $\mathrm{BDNF} / \mathrm{TrkB}$ signaling-related proteins ( $\mathrm{p}$ TrkB and BDNF) were significantly decreased in CLP mice compared with control and sham mice $(p<0.05$, Fig. 2b). The treatment of Emodin markedly enhanced 

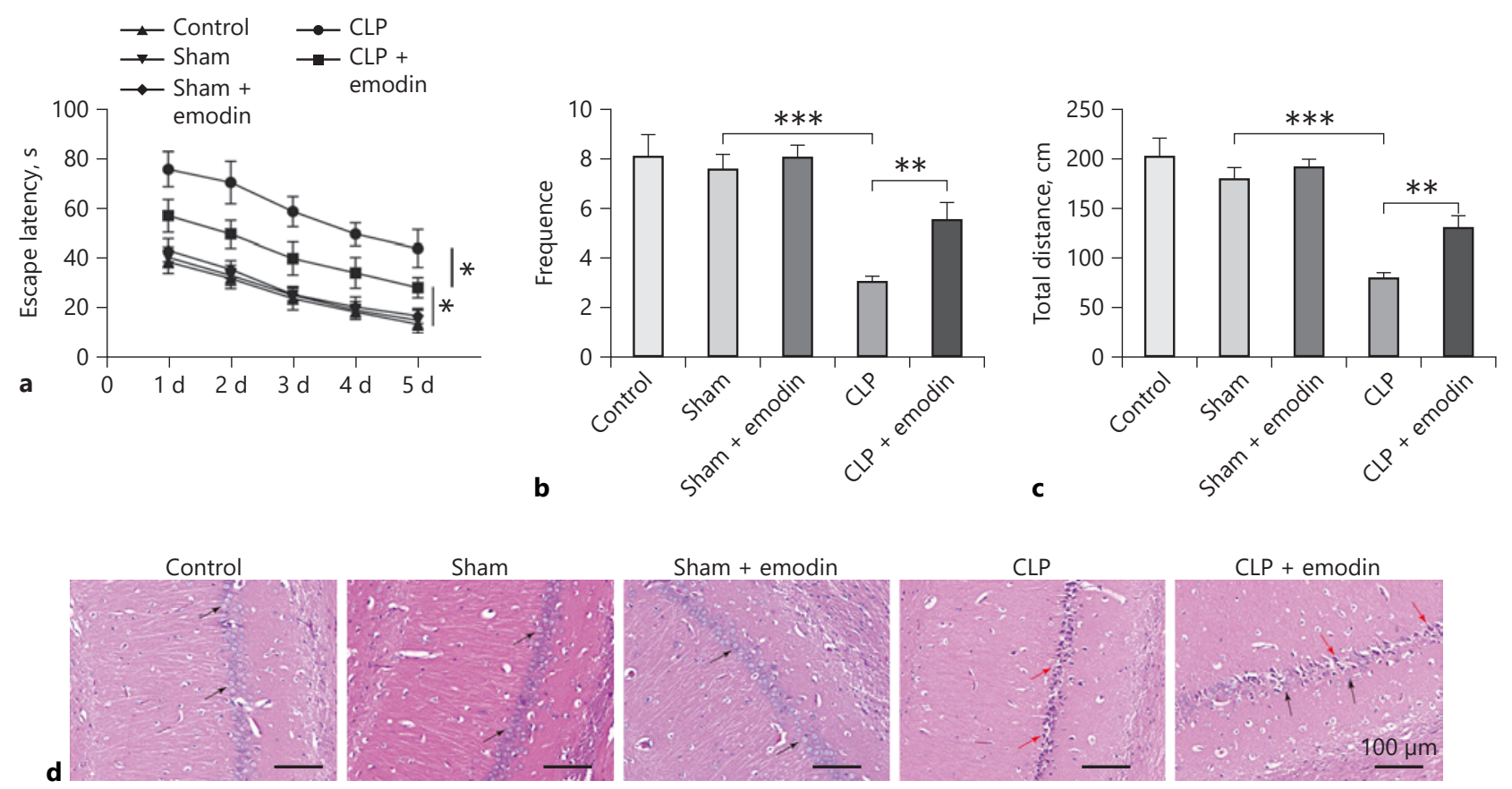

Fig. 1. Emodin improved cognitive dysfunction and suppressed pathological injury of hippocampal neurons in SAE mice. Escape latency (a) and frequency for mice crossing platform quadrant (b) in MWM were measured in different mice groups. c Movement distance in open field test in different mice groups was measured. d HE staining was conducted in hippocampal neurons in different groups of mice. The black arrow marks the normal neurons in the

the expression of $\operatorname{TrkB}, \mathrm{p}-\operatorname{TrkB}$, and BDNF which was decreased by CLP in SAE mice. These results showed that Emodin inhibited inflammatory factors and activated $\mathrm{BDNF} /$ TrkB signaling in CLP mice.

\section{Emodin Inhibited Apoptosis and Promoted Autophagy} of Hippocampal Neurons in SAE Mice

Previous reports have confirmed that Emodin is correlated with cell apoptosis and autophagy [26]; therefore, we detected the effect of Emodin on cell apoptosis and autophagy in SAE. As shown in Figure 3a, cell apoptosis of hippocampal neurons was significantly promoted when treated by CLP, which was remarkably rescued by treatment of Emodin (all $p<0.05$ ). CLP treatment obviously upregulated the levels of Bax, cleaved caspase-3, and inhibited the expression of Bcl-2 in mice, while Emodin reversed this phenomenon ( $p<0.05$, Fig. $3 b)$. It was reported that relatively to autophagy measurement, levels of LC3-II should be compared not to LC3-I but ideally to normal hippocampus tissue, and the red arrow marks the damaged neurons in the hippocampus tissue of CLP rats. Each group had 6 mice. All experiments were repeated in triplicate. ${ }^{*} p<0.05,{ }^{* *} p<$ $0.01,{ }^{* * *} p<0.001$. MWM, Morris water maze; HE, hematoxylin and eosin; SAE, sepsis-associated encephalopathy; CLP, cecal ligation and puncture.

more than one "housekeeping" protein and LC3 assays should be evaluated with and without autophagy inhibitor (3-MA) [27]. Therefore, the ratio of LC3-II/ $\beta$-actin and LC3-II/GAPDH was detected. The result revealed the ratio of LC3 II/GAPDH and LC3-II/ $\beta$-actin, and levels of Beclin-1 were markedly downregulated in CLP mice compared with control and sham groups, which was reversed by Emodin ( $p<0.05$, Fig. 3c). However, the effect of Emodin on these proteins was restored by 3-MA ( $p<$ 0.05 , Fig. $3 c$ ). All these results suggested that Emodin suppressed cell apoptosis and promoted autophagy in hippocampal neurons in SAE mice.

\section{Emodin Improved Cognitive Dysfunction and}

Suppressed Pathological Injury and Inflammatory

Factors through Activation of BDNF/TrkB Signaling in SAE Mice

To further explore the mechanism by which Emodin mediates the progression of SAE, BDNF/TrkB signaling 

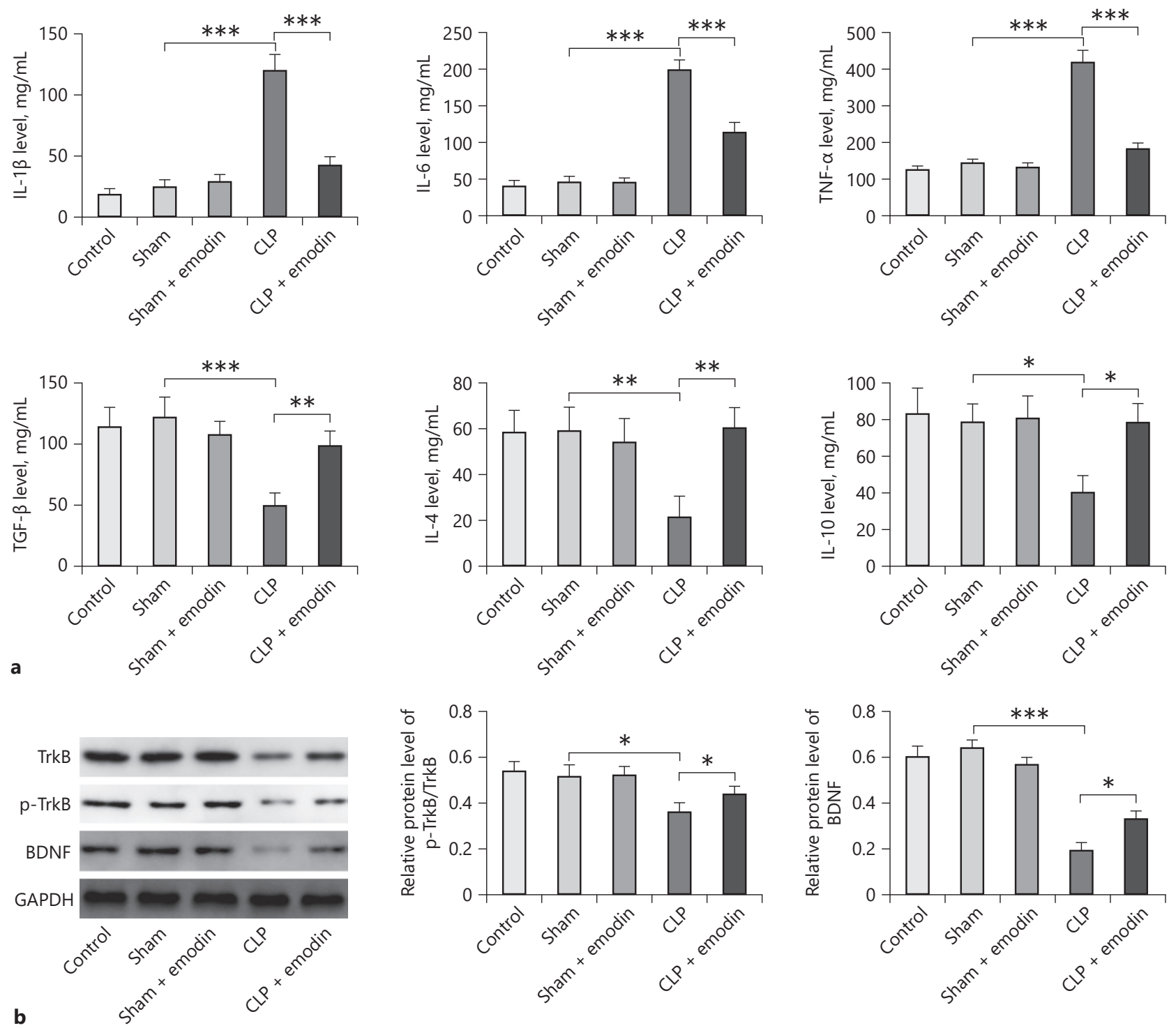

Fig. 2. Emodin inhibited levels of inflammatory factors and activated BDNF/TrkB signaling in SAE mice. a Pro-inflammatory factors of IL- $1 \beta$, IL- 6 , TNF- $\alpha$, and anti-inflammatory factors of IL-10, IL- 4 , and TGF- $\beta$ were measured by ELISA in different groups of mice. b Protein levels of TrkB, p-TrkB, and BDNF were evaluated by Western blotting in different mice groups. Each group had 6 mice. All experiments were repeated in triplicate. ${ }^{*} p<0.05,{ }^{* *} p<$ $0.01,{ }^{* * *} p<0.001$. ELISA, enzyme-linked immunosorbent assay; SAE, sepsis-associated encephalopathy; CLP, cecal ligation and puncture. inhibitor K252a was used to treat the mice. As revealed in Figure $4 a-c$, the escape latency in CLP-induced mice was significantly decreased by Emodin, while it was increased after adding K252a. In contrast, the frequency of crossing platform quadrant for CLP-induced mice was increased by Emodin, while it was reversed after adding K252a. Moreover, the movement distance of CLP-induced mice was also markedly increased by Emodin; however, it was decreased by K252a (all $p<0.05$ ).

Similarly, BDNF/TrkB signaling inhibitor K252a partially inhibited the protective effect of Emodin on hippocampal neuron injury (Fig. 4d). Emodin significantly decreased the levels of pro-inflammatory factors (IL-1 $\beta$, IL-6, and TNF- $\alpha$ ) and upregulated the levels of anti-in- 

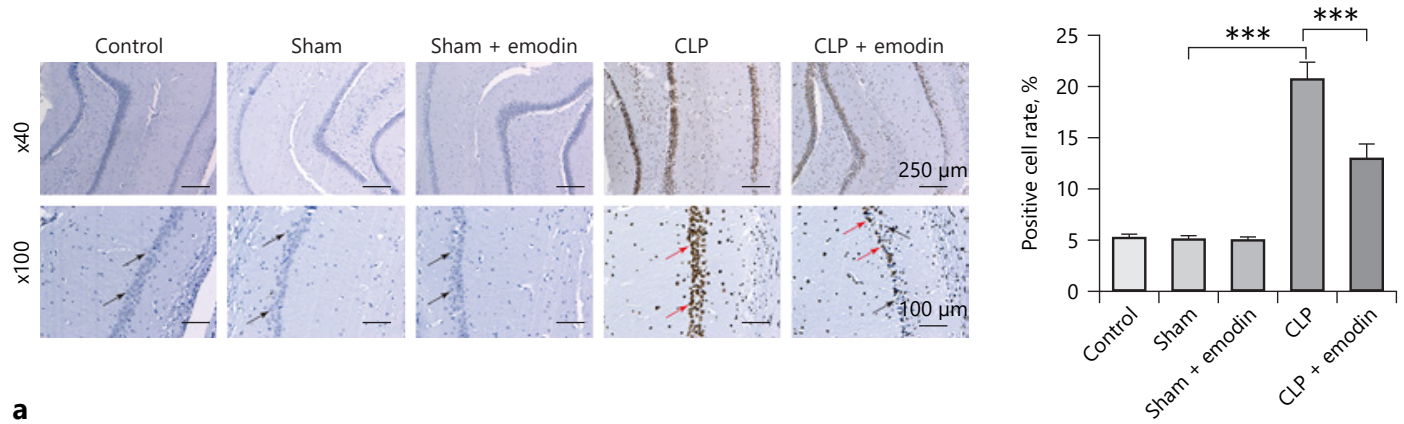

a
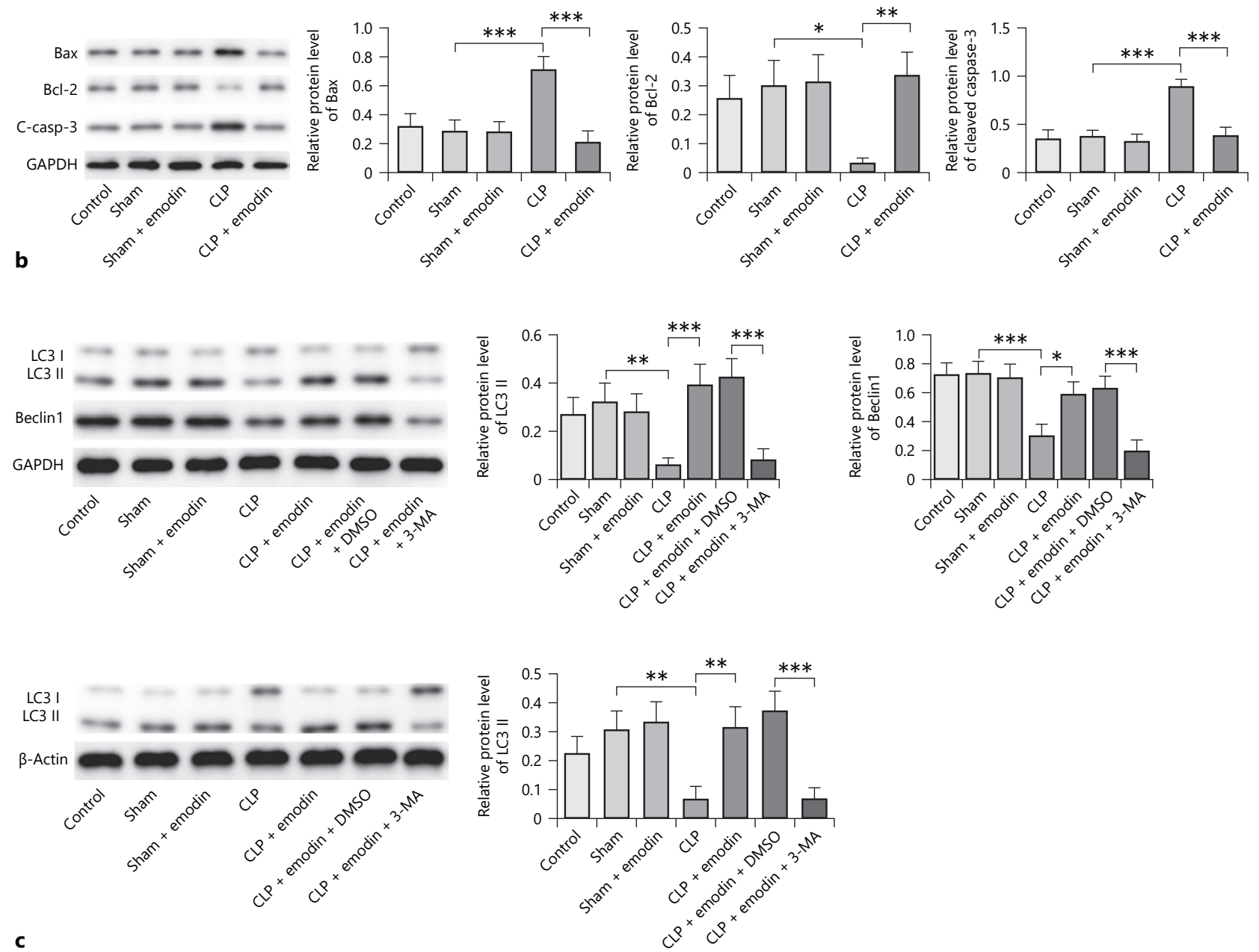

Fig. 3. Emodin inhibited apoptosis and promoted autophagy of hippocampal neurons in SAE mice. a Apoptosis of hippocampal neurons was measured by TUNEL assay in different mice groups. The blue cells (indicate the normal cells) were marked by black arrows, and the brown cells (indicate the apoptotic cells) were marked by red arrows. b Protein levels of Bcl-2, Bax, and cleaved caspase- 3 were detected by Western blot. c Western blot analyses showing LC3-II, LC3-I, and Beclin-1 levels and quantitative representation of LC3-II and Beclin-1 to GAPDH ratio. Each group had 6 mice. All experiments were repeated in triplicate. ${ }^{*} p<0.05$, ${ }^{* *} p<0.01,{ }^{* * *} p<0.001$. SAE, sepsis-associated encephalopathy; CLP, cecal ligation and puncture. 

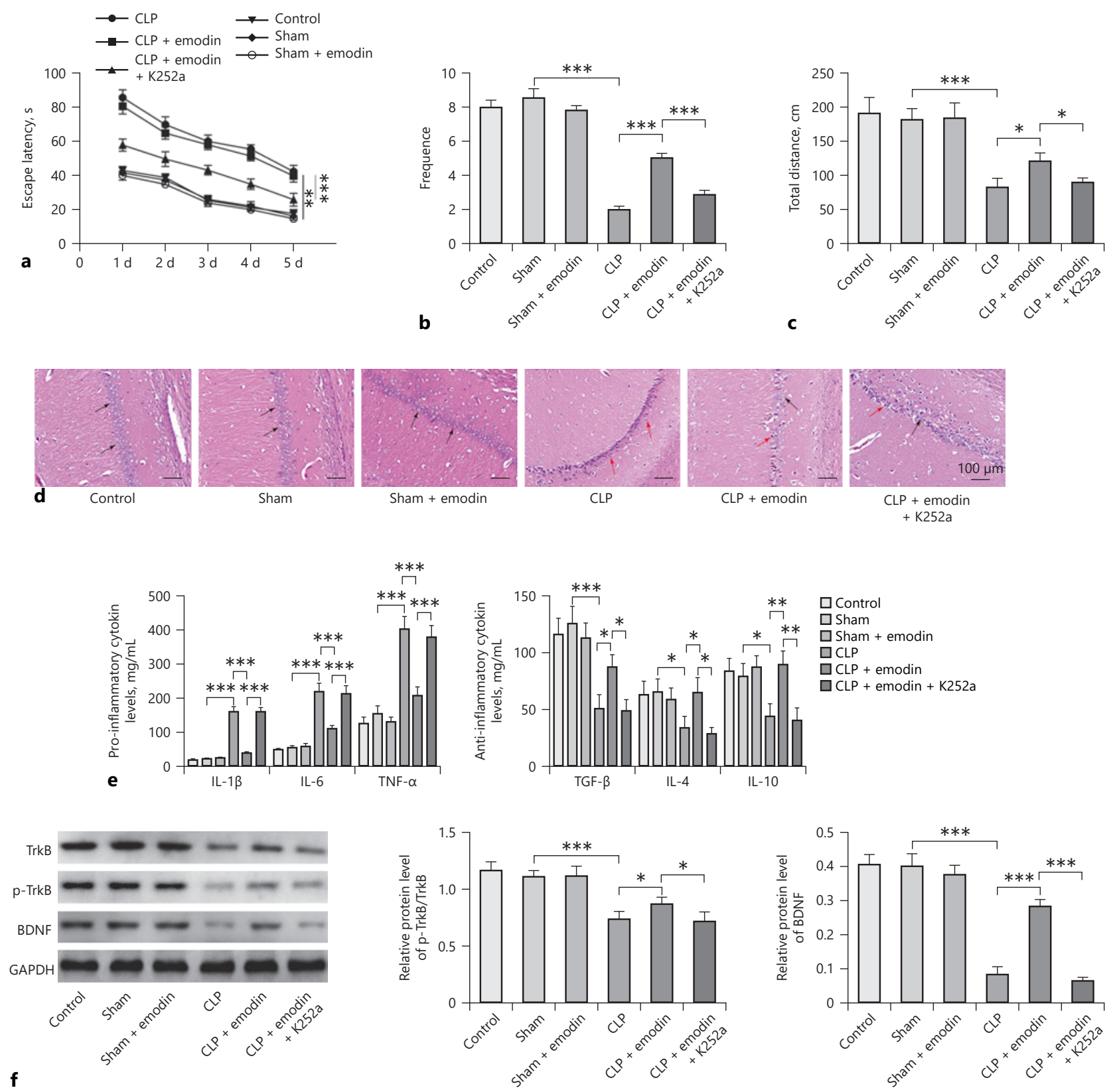

Fig. 4. Emodin improved cognitive dysfunction and suppressed pathological injury and inflammatory factors through activation of BDNF/TrkB signaling in SAE mice. Escape latency (a) and frequency for mice crossing platform quadrant (b) in MWM were measured in different mice groups. c Movement distance in open field test in different mice groups was measured. $\mathbf{d}$ HE staining was conducted in hippocampal neurons in different groups of mice. e Pro-inflammatory factors (IL-1 $\beta$, IL-6, and TNF- $\alpha$ ) and anti- inflammatory factors (IL-10, IL-4, and TGF- $\beta$ ) were measured by ELISA in different groups of mice. $\mathbf{f}$ Protein levels of TrkB, $p$-TrkB, and BDNF were evaluated by Western blotting in different mice groups. Each group had 6 mice. All experiments were repeated in triplicate. ${ }^{*} p<0.05,{ }^{* *} p<0.01,{ }^{* * *} p<0.001$. MWM, Morris water maze; HE, hematoxylin and eosin; ELISA, enzyme-linked immunosorbent assay; SAE, sepsis-associated encephalopathy; CLP, cecal ligation and puncture. 


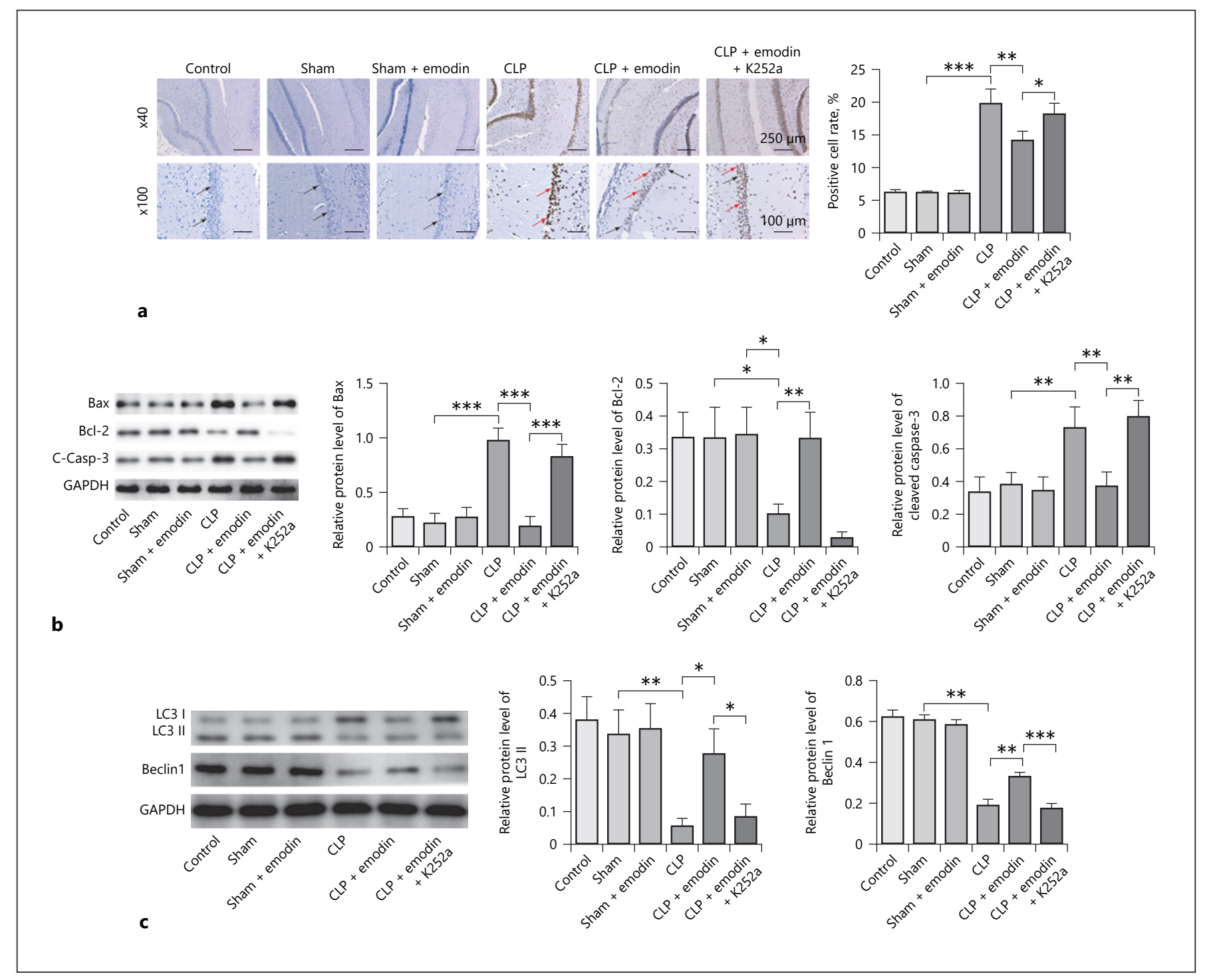

Fig. 5. Emodin inhibited apoptosis and promoted autophagy of hippocampal neurons through activation of BDNF/TrkB signaling in SAE mice. a Apoptosis of hippocampal neurons was measured by TUNEL assay in different mice groups. b Protein levels of Bcl-2, Bax, and caspase- 3 were detected by Western blot. c Western blot

flammatory factors (IL-10, IL-4, and TGF- $\beta$ ) in CLPtreated mice. However, these phenomena were significantly reversed by K252a (all $p<0.05$, Fig. 4e). Protein levels of TrkB, p-TrkB, and BDNF in CLP mice were increased by Emodin, while they were decreased in the presence of K252a (all $p<0.05$, Fig. 4f). All these results indicated that inhibition of $\mathrm{BDNF} / \mathrm{TrkB}$ signaling reversed Emodin-induced alleviation of cognitive dysfunction and pathological injury in SAE mice. analyses showing LC3-II, LC3-I, and Beclin-1 levels and quantitative representation of LC3-II and Beclin-1 to GAPDH ratio. Each group had 6 mice. All experiments were repeated in triplicate. ${ }^{*} p$ $<0.05,{ }^{* *} p<0.01,{ }^{* * *} p<0.001$. SAE, sepsis-associated encephalopathy; CLP, cecal ligation and puncture.

Emodin Inhibited Apoptosis and Promoted Autophagy of Hippocampal Neurons through Activation of

BDNF/TrkB Signaling in SAE Mice

Moreover, we found that cell apoptosis was markedly decreased in CLP mice after adding Emodin, while the anti-apoptotic effect of Emodin was reversed by K252a $(p<0.05$, Fig. 5a). After adding Emodin, the expressions of cleaved caspase- 3 and Bax were decreased and the level of Bcl-2 was increased in CLP mice, while it was significantly restored by K252a (all $p<0.05$, Fig. 5b). Be- 
sides, ratio of LC3 II/GAPDH, as well as protein levels of Beclin-1, was markedly enhanced by treatment of Emodin; however, co-treatment of K252a significantly reversed the effects (all $p<0.05$, Fig. $5 \mathrm{c}$ ). All these results suggested that inhibition of BDNF/TrkB signaling could reverse Emodin-induced inhibition of apoptosis and promotion of autophagy in hippocampal neurons of SAE mice.

\section{Discussion}

SAE is one of the most common complications for sepsis, developing in almost $70 \%$ sepsis patients with a high mortality rate [22]. Despite the high incidence and mortality rate in sepsis patients, treatment methods for SAE are still inadequate and its molecular mechanisms keep unclear. In the present study, we demonstrated for the first time that Emodin improved cognitive dysfunction, suppressed pathological injury and inflammation, as well as inhibited apoptosis and promoted autophagy through activation of BDNF/TrkB signaling in SAE mice.

The alteration of cognitive dysfunction is one of the severe sequences of SAE. It was widely found SAE patients and animals might develop cognitive dysfunction $[28,29]$. However, clinical strategy for improving the damaged cognitive function is still lacking. Zeng et al. [30] demonstrated that Emodin improved hyperhomocysteinemia-induced dementia and Alzheimer's diseaselike features in rat model. However, the effects of Emodin on cognitive dysfunction are largely unknown. In this research, we found for the first time that Emodin improved CLP-induced cognitive dysfunction in SAE mice.

Effects of Emodin on inflammation and cell apoptosis have been already reported in several researches. In an early study, it was found that Emodin could improve ischemic myocardium by inhibition of inflammation and cell apoptosis [31]. Another research also demonstrated that Emodin attenuated LPS-induced apoptosis and inflammation in ATDC5 cells [13]. On the other hand, it has been reported that the progression of SAE could lead to the secretion of pro-inflammatory cytokines $[27,32]$. In addition, IL- $1 \beta$, IL- 6 , and TNF- $\alpha$ levels were upregulated during the development of SAE [32]. Thus, these 3 cytokines were selected for analysis in this study. Meanwhile, it has been reported that anti-inflammatory and pro-inflammatory cytokines often complement each other in the process of inflammation and infection $[33,34]$. In this research, we found that CLPinduced upregulation of pro-inflammatory cytokines

Emodin Improves Sepsis-Associated Encephalopathy and downregulation of anti-inflammatory cytokines were reversed by Emodin. Thus, our study was in line with the previous data $[34,35]$. Thus, it can be demonstrated that Emodin inhibited inflammation and cell apoptosis in SAE mice.

The activation effects of Emodin on cell autophagy are also noticed in many researches. It was found Emodin could reduce cisplatin-induced apoptosis of rat renal tubular cells by activation of autophagy [36]. In lung cancer, it was also demonstrated that Emodin activates autophagy-related cell death [37]. Despite these researches, this is the first time we showed Emodin also activated cell autophagy in SAE mice. On the other hand, LC3 (LC3-I and LC3-II) and Beclin-1 are known to be involved in cell autophagy [38]. In addition, LC3 and Beclin-1 upregulation can lead to the formation of autophagosome [39]. Meanwhile, LC3I is usually converted to LC3II during the formation of autophagosome [40]. More importantly, according to the previous works [41, 42], levels of LC3-II should be compared not to LC3-I but ideally to more than one "housekeeping" protein in autophagy detection and LC3 assays should be evaluated with and without autophagy inhibitor (3-MA) [27]. As expected, CLP significantly inhibited the ratio of LC3-II/GAPDH and Beclin-1 level, and this effect was abolished by Emodin. Nevertheless, the effect of Emodin was restored by 3-MA. Thus, it can be concluded that Emodin induced the autophagy in CLP-induced mice through mediation of LC3 and Beclin-1.

MWM was regarded to represent the ability of learning and memory processes [24]. In addition, the increase of escape latency means the loss of memory capacity [24]. Our finding revealed that CLP-induced upregulation of escape latency was reversed by Emodin. Thus, Emodin could act as a novel agent for the treatment of SAE. On the other hand, BDNF/TrkB signaling was found to play important roles in SAE and other cognitive dysfunctions. $\mathrm{Xu}$ et al. [43] demonstrated that the activation of BDNF/ TrkB signaling led to improvement of SAE. Another study also found valproic acid could improve cognitive dysfunction in SAE by activating BDNF/TrkB signaling [44]. On the contrary, inhibition of BDNF/TrkB signaling resulted in acceleration of cognitive dysfunction in aging mice [45]. Therefore, it can be concluded that Emodin could decrease the escape latency in CLP mice via mediation of BDNF/TrkB signaling. Frankly speaking, the correlation between Emodin and BDNF/TrkB signaling has been unclear. In this research, we showed for the first time that Emodin also improves SAE through activation of $\mathrm{BDNF} /$ TrkB signaling. 
In conclusion, this in vivo study used a mice model to demonstrate that Emodin improved cognitive dysfunction, suppressed pathological injury and inflammation, as well as inhibited apoptosis and promoted autophagy through activation of BDNF/TrkB signaling in SAE mice. This study might provide basic and experimental evidences for Emodin in treatment of SAE.

\section{Statement of Ethics}

The whole animal study was approved by the Experimental Animal Ethics Committee at the First Hospital of Jilin University (approval number 20200009).

\section{Conflict of Interest Statement}

No conflicts of interest, financial or otherwise, are declared by the authors.

\section{Funding Sources}

This work was supported by Jilin Province Science and Technology Development Plan Project (20200201319JC).

\section{Author Contributions}

Li-Li Gao performed concepts, design, funding acquisition, experimental studies, and writing of original draft preparation; ZhiHao Wang conceived data acquisition and data analysis; Yu-Hang $\mathrm{Mu}$ involved in experimental studies; Zuo-Long Liu performed design, data analysis, and supervision; Li Pang performed concepts, design, supervision, writing, reviewing, and editing; all the authors approved for the final version.

\section{Data Availability Statement}

All data generated or analyzed during this study are included in this article. Further enquiries can be directed to the corresponding author.

\section{References}

1 Heming N, Mazeraud A, Verdonk F, Bozza FA, Chrétien F, Sharshar T. Neuroanatomy of sepsis-associated encephalopathy. Crit Care. 2017;21(1):65-6.

2 Sonneville R, de Montmollin E, Poujade J, Garrouste-Orgeas M, Souweine B, Darmon $\mathrm{M}$, et al. Potentially modifiable factors contributing to sepsis-associated encephalopathy. Intensive Care Med. 2017;43(8):1075-84.

3 Feng Q, Ai YH, Gong H, Wu L, Ai ML, Deng SY, et al. Characterization of sepsis and sepsis-associated encephalopathy. J Intensive Care Med. 2019 Nov-Dec;34(11-12):93845.

4 Ren C, Yao RQ, Zhang H, Feng YW, Yao YM. Sepsis-associated encephalopathy: a vicious cycle of immunosuppression. J Neuroinflammation. 2020;17(1):14-5.

5 Jin P, Deng S, Tian M, Lenahan C, Wei P, Wang Y, et al. INT-777 prevents cognitive impairment through activating Takeda G protein-coupled receptor 5 (TGR5) by attenuating neuroinflammation via cAMP/PKA/ CREB signaling axis in a rat model of sepsis. Exp Neurol. 2021;335:113504.

6 Mei B, Li J, Zuo Z. Dexmedetomidine attenuates sepsis-associated inflammation and encephalopathy via central alpha2A adrenoceptor. Brain Behav Immun. 2021;91:296-314.

7 Lu G, Liu H, Li D, Qiao L, Liu Z, Ma Z. Fournier gangrene caused by Escherichia coli complicated with septic shock and sepsis-associated encephalopathy in an 8-month-old girl: a case report. Minerva Med. 2020. Epub ahead of print.

8 Wen Y, Han C, Liu T, Wang R, Cai W, Yang $\mathrm{J}$, et al. Chaiqin chengqi decoction alleviates severity of acute pancreatitis via inhibition of TLR4 and NLRP3 inflammasome: identification of bioactive ingredients via pharmacological sub-network analysis and experimental validation. Phytomedicine. 2020;79: 153328.

9 Tu YJ, Tan B, Jiang L, Wu ZH, Yu HJ, Li XQ, et al. Emodin inhibits lipopolysaccharide-induced inflammation by activating autophagy in RAW 264.7 cells. Chin J Integr Med. 2021; 27(5):345-52.

10 Zhu T, Zhang W, Feng S-J, Yu H-P. Emodin suppresses LPS-induced inflammation in RAW264.7 cells through a PPAR $\gamma$-dependent pathway. Int Immunopharmacol. 2016;34: 16-24.

11 Han JW, Shim DW, Shin WY, Heo KH, Kwak SB, Sim EJ, et al. Anti-inflammatory effect of emodin via attenuation of NLRP3 inflammasome activation. Int J Mol Sci. 2015;16(4): 8102-9.

12 Xia S, Ni Y, Zhou Q, Liu H, Xiang H, Sui H, et al. Emodin attenuates severe acute pancreatitis via antioxidant and anti-inflammatory activity. Inflammation. 2019;42(6):2129-38.

13 Liang Z, Ren C. Emodin attenuates apoptosis and inflammation induced by LPS through up-regulating lncRNA TUG1 in murine chondrogenic ATDC5 cells. Biomed Pharmacother. 2018;103:897-902.

14 Tao X, Yan M, Wang L, Zhou Y, Wang Z, Xia $\mathrm{T}$, et al. Effects of estrogen deprivation on memory and expression of related proteins in ovariectomized mice. Ann Transl Med. 2020; 8(6):356.

15 Guo R, Li Y, Han M, Liu J, Sun Y. Emodin attenuates acute lung injury in Cecal-ligation and puncture rats. Int Immunopharmacol. 2020;85:106626.

16 Li Y, Guo R, Zhang M, Chen P, Li J, Sun Y. Protective effect of emodin on intestinal epithelial tight junction barrier integrity in rats with sepsis induced by cecal ligation and puncture. Exp Ther Med. 2020;19(6):352130.

17 Song X, Liu B, Cui L, Zhou B, Liu W, Xu F, et al. Silibinin ameliorates anxiety/depressionlike behaviors in amyloid $\beta$-treated rats by upregulating $\mathrm{BDNF} / \mathrm{TrkB}$ pathway and attenuating autophagy in hippocampus. Physiol Behav. 2017;179:487-93.

18 Sun X, Zhang T, Zhao Y, Cai E, Zhu H, Liu S. The protective effect of 5-O-methylvisammioside on LPS-induced depression in mice by inhibiting the over activation of BV-2 microglia through Nf- $\kappa \mathrm{B} / \mathrm{I} \kappa \mathrm{B}-\alpha$ pathway. Phytomedicine. 2020;79:153348.

19 Ma Z, Liu K, Li XR, Wang C, Liu C, Yan DY, et al. Alpha-synuclein is involved in manganese-induced spatial memory and synaptic plasticity impairments via TrkB/Akt/Fynmediated phosphorylation of NMDA receptors. Cell Death Dis. 2020;11(10):834.

$20 \mathrm{Xu}$ XE, Liu L, Wang YC, Wang CT, Zheng Q, Liu QX, et al. Caspase-1 inhibitor exerts brain-protective effects against sepsis-associated encephalopathy and cognitive impairments in a mouse model of sepsis. Brain Behav Immun. 2019;80:859-70.

21 Xu XE, Li MZ, Yao ES, Gong S, Xie J, Gao W, et al. Morin exerts protective effects on encephalopathy and sepsis-associated cognitive functions in a murine sepsis model. Brain Res Bull. 2020;159:53-60. 
22 Tian M, Qingzhen L, Zhiyang Y, Chunlong C, Jiao D, Zhang L, et al. Attractylone attenuates sepsis-associated encephalopathy and cognitive dysfunction by inhibiting microglial activation and neuroinflammation. J Cell Biochem. 2019;120(5):7101-8.

23 Li XY, Zhang YQ, Xu G, Li SH, Li H. miR-124/ MCP-1 signaling pathway modulates the protective effect of itraconazole on acute kidney injury in a mouse model of disseminated candidiasis. Int J Mol Med. 2018;41(6):3468-76.

24 Saini S, Sharma T, Jain A, Kaur H, Katare OP, Singh B. Systematically designed chitosancoated solid lipid nanoparticles of ferulic acid for effective management of Alzheimer's disease: a preclinical evidence. Colloids Surf B Biointerfaces. 2021;205:111838.

25 He YQ, Zhou CC, Yu LY, Wang L, Deng JL, Tao YL, et al. Natural product derived phytochemicals in managing acute lung injury by multiple mechanisms. Pharmacol Res. 2021; 163:105224.

26 Shen F, Ge C, Yuan P. Aloe-emodin induces autophagy and apoptotic cell death in nonsmall cell lung cancer cells via Akt/mTOR and MAPK signaling. Eur J Pharmacol. 2020;886: 173550.

27 Klionsky DJ, Abdel-Aziz AK, Abdelfatah S, Abdellatif M, Abdoli A, Abel S, et al. Guidelines for the use and interpretation of assays for monitoring autophagy (4th edition)(1). Autophagy. 2021;17(1):1-382.

28 Andonegui G, Zelinski EL, Schubert CL, Knight D, Craig LA, Winston BW, et al. Targeting inflammatory monocytes in sepsis-associated encephalopathy and long-term cognitive impairment. JCI insight. 2018;3(9):e99364.

29 Sun F, Si Y, Bao H, Xu Y, Pan X, Zeng L, et al. Regulation of sirtuin 3-mediated deacetylation of cyclophilin D attenuated cognitive dysfunction induced by sepsis-associated encephalopathy in mice. Cell Mol Neurobiol. 2017;37(8):1457-64.

30 Zeng P, Shi Y, Wang XM, Lin L, Du YJ, Tang $\mathrm{N}$, et al. Emodin rescued hyperhomocystein- emia-induced dementia and Alzheimer's disease-like features in rats. Int J Neuropsychopharmacol. 2019;22(1):57-70.

31 Wu Y, Tu X, Lin G, Xia H, Huang H, Wan J, et al. Emodin-mediated protection from acute myocardial infarction via inhibition of inflammation and apoptosis in local ischemic myocardium. Life Sci. 2007;81(17-18):13328.

32 Xie J, Zhao ZZ, Li P, Zhu CL, Guo Y, Wang J, et al. Senkyunolide I protects against sepsisassociated encephalopathy by attenuating sleep deprivation in a murine model of cecal ligation and puncture. Oxid Med Cell Longev. 2021;2021:6647258.

33 Li S, Guo H, Xu X, Hua R, Zhao Q, Li J, et al. Therapeutic methods for gut microbiota modification in lipopolysaccharide-associated encephalopathy. Shock. 2021;56(5):82431.

34 Yang C, Yang C, Huang Z, Zhang J, Chen N, Guo Y, et al. Reduced expression of MiR125a-5p aggravates LPS-induced experimental acute kidney injury pathology by targeting TRAF6. Life Sci. 2021:119657. Epub ahead of print.

35 Sun Y, Wang C, Zhang N, Liu F. Melatonin ameliorates hypertension in hypertensive pregnant mice and suppresses the hypertension-induced decrease in $\mathrm{Ca}(2+)$-activated $\mathrm{K}(+)$ channels in uterine arteries. Hypertens Res. 2021;44(9):1079-86.

36 Liu H, Gu LB, Tu Y, Hu H, Huang YR, Sun W. Emodin ameliorates cisplatin-induced apoptosis of rat renal tubular cells in vitro by activating autophagy. Acta Pharmacol Sin. 2016;37(2):235-45.

37 Haque E, Kamil M, Irfan S, Sheikh S, Hasan A, Nazir A, et al. Blocking mutation independent $\mathrm{p} 53$ aggregation by emodin modulates autophagic cell death pathway in lung cancer. Int J Biochem Cell Biol. 2018;96:905.

38 Hseu YC, Vudhya Gowrisankar Y, Wang LW, Zhang YZ, Chen XZ, Huang PJ, et al. The in vitro and in vivo depigmenting activity of pterostilbene through induction of autophagy in melanocytes and inhibition of UVA-irradiated $\alpha-\mathrm{MSH}$ in keratinocytes via Nrf2-mediated antioxidant pathways. Redox Biol. 2021; 44:102007.

39 Fu SC, Lin JW, Liu JM, Liu SH, Fang KM, Su $\mathrm{CC}$, et al. Arsenic induces autophagy-dependent apoptosis via Akt inactivation and AMPK activation signaling pathways leading to neuronal cell death. Neurotoxicology. 2021;85:133-44.

40 Lin J, Ruan J, Zhu H, Chen Z, Chen J, Yu H. Tenacissoside $\mathrm{H}$ induces autophagy and radiosensitivity of hepatocellular carcinoma cells by $\mathrm{PI} 3 \mathrm{~K} / \mathrm{Akt} / \mathrm{mTOR}$ signaling pathway. Dose Response. 2021 Apr-Jun;19(2): 15593258211011023.

41 Peng H, Liu B, Yves TD, He Y, Wang S, Tang $\mathrm{H}$, et al. Zika virus induces autophagy in human umbilical vein endothelial cells. Viruses. 2018;10(5):259.

42 Liu P, He S, Gao J, Li J, Fan X, Xiao YB. Liver $\mathrm{X}$ receptor activation protects against inflammation and enhances autophagy in myocardium of neonatal mouse challenged by lipopolysaccharides. Biosci Biotechnol Biochem. 2014;78(9):1504-13.

43 Xu C, Yin X, Sun X, Xing J, Sun Y. Baicalein improves sepsis-associated encephalopathy via suppressing oxidative stress and iNOSmediated NO production and enhancing BDNF/TrkB signaling. Int J Clin Exp Med. 2016;9(2):1346-53.

$44 \mathrm{Wu}$ J, Dong L, Zhang M, Jia M, Zhang G, Qiu $\mathrm{L}$, et al. Class I histone deacetylase inhibitor valproic acid reverses cognitive deficits in a mouse model of septic encephalopathy. Neurochem Res. 2013;38(11):2440-9.

45 Qiu LL, Pan W, Luo D, Zhang GF, Zhou ZQ, Sun XY, et al. Dysregulation of BDNF/TrkB signaling mediated by NMDAR/Ca2+/calpain might contribute to postoperative cognitive dysfunction in aging mice. J Neuroinflammation. 2020;17(1):23-15. 